\title{
The complexity of the certification of properties of Stable Marriage
}

\author{
Daniel J. Dougherty and Stanley M. Selkow \\ Worcester Polytechnic Institute Worcester, MA 01609 USA
}

\begin{abstract}
We give some lower bounds on the certificate complexity of some problems concerning stable marriage, answering a question of Gusfield and Irving.
\end{abstract}

Key words: stable marriage, combinatorial problems, computational complexity, certificate complexity

\section{Introduction}

An $n$-instance of the stable marriage problem is given by two disjoint sets $\mathcal{M}$ and $\mathcal{W}$ of the same size $n$, conventionally called the men and the women, together with, for each person, a total order on the opposite sex. A matching, or marriage, $M$ is a one-to-one correspondence between $\mathcal{M}$ and $\mathcal{W}$. Man $m$ and woman $w$ are a blocking pair for a marriage $M$ if $m$ and $w$ are not partners in $M$ yet $w$ precedes $m$ 's partner on $m$ 's list and $m$ precedes $w$ 's partner on $w$ 's list. A marriage is stable if there are no blocking pairs.

Gale and Shapley [2] showed that any problem instance admits at least one stable marriage. There are algorithms to construct such marriages that run in $O\left(n^{2}\right)$ time; $\mathrm{Ng}$ and Hirschberg [4] have shown that this complexity is asymptotically optimal.

In their monograph [3] Gusfield and Irving include the following among the Open Problems: 
..it requires $\Omega\left(n^{2}\right)$ time to check whether a matching is stable. However, once instability is established, there is a very succinct certificate of instability, namely a blocking pair. Is there a succinct certificate $\left(\mathrm{o}\left(n^{2}\right)\right.$ size $)$ of stability?

In this note we give a negative answer to this question, and also give lower bounds for the certificate complexity of some related questions.

We encode an $n$-instance of a problem as follows. We may identify the set $\mathcal{M}$ of men and the set $\mathcal{W}$ of women each with the set $\{1, \ldots, n\}$, and represent each person's preference list as a permutation of $\{1, \ldots, n\}$, so a problem instance can be represented as a pair of $n \times n$ matrices of natural numbers.

Although formally we identify $\mathcal{M}$ and $\mathcal{W}$ with $\{1, \ldots, n\}$ it will often be clearer to use notation like " $m_{i}$ " to refer to man $i$. We will write $\rho_{m}$ [resp, $\rho_{w}$ ] for the permutation associated with man $m$ [resp., with woman $w$ ]. So for example $\rho_{m}(w)=k$ expresses the fact that the man numbered $m$ ranks the woman numbered $w$ as $k$ th on his preference list.

A pair $(m, w)$ in an instance $I$ is a stable pair if there is a stable marriage pairing $m$ with $w$, otherwise it is an unstable pair; $(m, w)$ is a fixed pair if every stable marriage for $I$ pairs $m$ and $w$.

Certificates There is a standard notion of "certificate complexity" in the literature, in the context of boolean functions [1]. Suppose $f:\{0,1\}^{n} \rightarrow\{0,1\}$. Let $C: S \rightarrow\{0,1\}$ be an assignment of values to some subset $S$ of the $n$ inputs; let the size of the certificate be the size of $S$. Say that $C$ is consistent with $x \in\{0,1\}^{n}$ if $x_{i}=C(i)$ for all $i \in S$. A 0 -certificate for $f$ is an assignment $C$ such that $f(x)=0$ whenever $x$ is consistent with $C$; a 1-certificate is defined similarly. The certificate complexity $C_{x}(f)$ of $f$ on $x$ is the size of a smallest $f(x)$-certificate that is consistent with $x$. The certificate complexity of $f$ is $\max _{x} C_{x}(f)$.

Stable matching is not directly presented in terms of boolean functions, but the following is the natural translation of certificate complexity to our setting.

Informally, a certificate $C$ is a partially specified instance. Formally, an $n$-certificate is a set of quadruples of the form $(\mathcal{M}, m, w, r)$ or $(\mathcal{W}, w, m, r)$ with $1 \leq m, w, r \leq n$. Here $r$ is interpreted as $\rho_{m}(w)$ in the preference matrix for $\mathcal{M}$ [or $\rho_{w}(m)$ in the matrix for $\mathcal{W}$, as appropriate]. The size of a certificate $C$ is the number of quadruples in $C$. 
A property is a family $\mathcal{P}=\left\{\mathcal{P}_{n} \mid n \in \omega\right\}$ where each $\mathcal{P}_{n}$ is a set of $n$-instances. For example, the property that $(m, w)$ IS A STABLE PAIR is the family of all instances in which pair $(m, w)$ is stable. If $I \in \mathcal{P}$ we may say that $I$ satisfies $\mathcal{P}$.

Let $\mathcal{P}$ be a property and let $I$ be a problem instance. The certificate $C$ witnesses $\mathcal{P}$ for $I$ if $I$ extends $C$ and every instance which extends $C$ satisfies $\mathcal{P}$. For example any certificate which includes $(\mathcal{M}, m, w, 1)$ and $(\mathcal{W}, w, m, 1)$ witnesses the property $(m, w)$ IS A FIXED PAIR (in any instance containing this data) since in any instance extending this certificate, $m$ and $w$ are each other's first choice.

The certificate complexity of $\mathcal{P}$ for $I$ is

$$
\mathrm{CC}(\mathcal{P}, I)=\min \{\operatorname{size}(C) \mid C \text { witnesses } \mathcal{P} \text { for } I\}
$$

The certificate complexity of $\mathcal{P}$ at $n$ is

$$
\mathrm{CC}(\mathscr{P}, n)=\max \{\mathrm{CC}(\mathscr{P}, I) \mid I \text { is an } n \text {-instance which satisfies } \mathscr{P}\}
$$

We write $\mathrm{CC}(\mathscr{P})$ for $\mathrm{CC}(\mathscr{P}, n)$ considered as a function of $n$.

If $M$ is an unstable marriage in an $n$-instance, then as Gusfield and Irving observe, in order to demonstrate that $M$ is unstable it suffices to exhibit a single blocking pair. That is, the certificate

$$
\left\{\left(\mathcal{M}, m, w, \rho_{m}(w)\right),\left(\mathcal{W}, w, m, \rho_{w}(m)\right),\left(\mathcal{M}, m, w^{\prime}, \rho_{m}\left(w^{\prime}\right)\right),\left(\mathcal{W}, w, m^{\prime}, \rho_{w}\left(m^{\prime}\right)\right)\right\}
$$

witnesses the instability of any marriage $M$ in which $\left(m, w^{\prime}\right),\left(m^{\prime}, w\right) \in M$ if $\rho_{m}(w)<\rho_{m}\left(w^{\prime}\right)$ and $\rho_{w}(m)<\rho_{w}\left(m^{\prime}\right)$. Since the size of this certificate does not grow with $n$ we may say that the certificate complexity of the property $M$ IS AN UNSTABLE MARRIAGE is constant.

\section{Lower bounds for certificate complexity}

Lemma 1 Let I be an instance such that each woman has the same preference ordering. Then I admits exactly one stable marriage.

Furthermore, if each woman ranks the men in the order $1,2, \ldots$, then each $m_{i}$ must be paired with the first $w$ in $m_{i}$ 's list such that $w$ is not paired with any $m_{s}$ for $s<i$. 
Proof. It suffices to prove the second assertion, since we may rename the men if necessary to satisfy the stronger hypothesis.

The proof is by induction on $n$. There is only one partner to whom man $m_{1}$ may be married: in every stable marriage he is paired with his top choice. If we then remove this pair from consideration we are left with a problem of size $n-1$ satisfying the hypothesis, so the result follows by induction.

Let $I_{n}^{*}$ denote the $n$-instance in which for every $m, m_{i} \in \mathcal{M}$ and every $w, w_{j} \in \mathcal{W}$, $\rho_{m}\left(w_{j}\right)=j$ and $\rho_{w}\left(m_{i}\right)=i$. Let $\operatorname{Id}_{n}$ be the marriage $\left\{\left(m_{1}, w_{1}\right), \ldots,\left(m_{n}, w_{n}\right)\right\}$.

It is easily seen that $I_{n}^{*}$ admits $\operatorname{Id}_{n}$ as its only stable marriage.

For $n \geq 3$ and $1 \leq j<k<l \leq n$, let $I^{j, k, l}$ be exactly the same as $I_{n}^{*}$ except that $w_{j}$ and $w_{l}$ are exchanged in $m_{k}$ 's preference list. That is, in $I^{j, k, l}$

$$
\begin{array}{ll}
\rho_{w}\left(m_{s}\right)=s & \text { for all } w \\
\rho_{m_{k}}\left(w_{j}\right)=l & \\
\rho_{m_{k}}\left(w_{l}\right)=j & \\
\rho_{m_{k}}\left(w_{i}\right)=i & \text { for } i \neq j, i \neq l . \\
\rho_{m}\left(w_{t}\right)=t & \text { for } m \neq m_{k} .
\end{array}
$$

Lemma 2 The instance $I^{j, k, l}$ admits exactly one stable marriage:

$\left\{\left(m_{i}, w_{i}\right) \mid 1 \leq i<k \vee l<i \leq n\right\} \cup\left\{\left(m_{k}, w_{l}\right\} \cup\left\{\left(m_{i}, w_{i-1}\right) \mid k<i \leq l\right\}\right.$.

Proof. This is a direct consequence of Lemma 1.

Let Id IS STABLE be the property defined, at each $n$, to be the set of $n$-instances for which $\operatorname{Id}_{n}$ is stable.

Theorem 3 The certificate complexity of the property Id IS STABLE is $\Omega\left(n^{2}\right)$.

Proof. We show that

$$
\mathrm{CC}\left(\mathrm{Id} \text { IS STABLE, } I_{n}^{*}\right) \text { is } \Omega\left(n^{2}\right) \text {. }
$$

It suffices to establish the claim that if $C$ is a certificate of the stability of Id for $I_{n}^{*}$ 
then for every $k$, either

$$
\forall j<k \quad\left(\mathcal{M}, m_{k}, w_{j}, j\right) \in C \quad \text { or } \quad \forall l>k \quad\left(\mathcal{M}, m_{k}, w_{l}, l\right) \in C
$$

since this obviously requires $\Omega\left(n^{2}\right)$ quadruples.

To verify the claim note that if it failed at $k$ we would have $\left(\mathcal{M}, m_{k}, w_{j}, j\right) \notin C$ for some $j<k$ and $\left(\mathcal{M}, m_{k}, w_{l}, l\right) \notin C$ for some $l>k$, so that $I^{j, k, l}$ would extend $C$. Since Id is not stable in $I^{j, k, l}$ this contradicts the the assumption that $C$ witnesses the stability of Id.

One might reasonably expect that there exist short certificates for stability properties of a single pair in an instance. But in fact the argument above shows that this is not the case.

Corollary 4 For any man-woman pair p, the certificate complexity of each of the following properties is $\Omega\left(n^{2}\right)$.

(1) $p$ IS A STABLE PAIR

(2) $p$ IS A FIXED PAIR

(3) $p$ IS AN UNSTABLE PAIR

Proof. For the first assertion we observe that the argument of Theorem 3 actually shows that in order that a certificate $C$ witness the stability of the pair $\left(m_{e}, w_{e}\right)$ in $I_{n}^{*}$ it is necessary that for every $k<e$,

$$
\forall j<k \quad\left(\mathcal{M}, m_{k}, w_{j}, j\right) \in C \quad \text { or } \quad \forall l>e \quad\left(\mathcal{M}, m_{k}, w_{l}, l\right) \in C .
$$

Since: if this were violated at $k$ we would have $\left(\mathcal{M}, m_{k}, w_{j}, j\right) \notin C$ for some $j<k$ and $\left(\mathcal{M}, m_{k}, w_{l}, l\right) \notin C$ for some $l \geq e$, so that $I^{j, k, l}$ would extend $C$. And by Lemma 2 the pair $\left(m_{e}, w_{e}\right)$ is not stable in $I^{j, k, l}$ (since $k<e \leq l$ ). So when $e=\lceil n / 2\rceil, C$ requires $\Omega\left(n^{2}\right)$ quadruples.

For the second assertion we simply note that since the instances $I_{n}^{*}$ admit a single stable marriage, a pair $p$ is stable in $I_{n}^{*}$ if and only if it is fixed. So the result follows from the previous part.

For the third assertion we note that in the instance $I_{n}^{*}$ the pair $p=\left(m_{\lceil n / 2\rceil}, w_{\lceil n / 2\rceil-1}\right)$ is unstable, but becomes stable if the values of any one pair $1 \leq j \leq k<n / 2 \leq l \leq n$ are exchanged. 


\section{References}

[1] H. Buhrman and R. de Wolf. Complexity measures and decision tree complexity: A survey. Theoretical Computer Science, 288:21-43, 2002.

[2] D. Gale and L Shapley. College admissions and the stability of marriage. American Mathematical Monthly, 69:9-15, 1962.

[3] D. Gusfield and R. W. Irving. The Stable Marriage Problem: Structure and Algorithms. MIT_Press, 1989.

[4] C. Ng and D. Hirschberg. Lower bounds for the stable marriage problem and its variants. SIAM J. Computing, 19:71-77, 1990. 